\title{
CORRESPONDENCE.
}

\section{ON SOME PRACTICAL APPLICATIONS OF SIMPLE INTERPOLATION FORMULZ.}

To the Editor of the Jownal of the Institute of Actuaries.

Sin,-The recent publication, by the Institute of Actuaries, of Joint-life Annuity values according to the experience of the Government Life Annuitants, tabulated, as regards one of the two lives involved, for quinquennial intervals of age only, has directed my attention to some simple practical applications of well-known interpolation formulæ, which may be of interest to Students of the Institute, and generally to those employing the tables in question.

Let there be given the following suecessive values, at quinquennial intervals, of any tabular function of two independent variables $x$ and $y$, so that

$$
\begin{aligned}
\phi_{x, y} & =a, & \phi_{x+5, y} & =c, \\
\phi_{x, y+5} & =b, & \phi_{x+5, y+5} & =d,
\end{aligned}
$$

and let it be required to deduce an approximate value for $\phi_{x+m . y+n}$, where $m$ and $n$ are each less than 5 .

We have, interpolating to first differences only,

$$
\begin{aligned}
\phi_{x, y+n} & =\phi_{x, y}+\frac{n}{5}\left(\phi_{x, y+5}-\phi_{x y}\right) \\
& =\frac{1}{5}\{(5-n) a+n b\} . . . . .
\end{aligned}
$$

Similarly, $\quad \phi_{x+5 . y+n}=\frac{1}{5}\{(5-n) e+n d\} . . . . .$.

whence we may deduce

$$
\begin{aligned}
\phi_{x+m, y+n} & =\phi_{x, y+n}+\frac{m}{5}\left(\phi_{x+5, y+n}-\phi_{x, y+n}\right) \\
& =\frac{1}{5}\left\{(5-m) \phi_{x, y+n}+m \phi_{x+5 . y+n}\right\} .
\end{aligned}
$$

and inserting the corresponding values from formulæ (1) and (2), and reducing, we have finally

$$
\phi_{x+m \cdot y+n}=\frac{1}{25}\{(5-m)(5-n) a+n(5-m) b+m(5-n) c+m n d .
$$

an expression which gives the approximate value of the function sought in terms of the values of the four given tabular functions instead of their differences, and which will sometimes be found in practice more convenient than the usual formula involving the differences of the functions. 
The following table gives the values of the multipliers of $a, b, c$, and $d$ respectively, for all values of $m$ and $n$ from 0 to 4 inclusive:

Given $\phi_{x . y}=a, \phi_{x . y+5}=b, \phi_{x+5 . y}=c, \phi_{x+5 . y+5}=d$.

To find $\phi_{x+m, y+n}(m$ and $n$ each $<5$ ).

\begin{tabular}{|c|c|c|c|c|c|c|c|c|c|c|c|c|c|c|c|c|c|c|c|c|}
\hline \multirow{3}{*}{$\begin{array}{c}\text { Values } \\
\text { of } n\end{array}$} & \multicolumn{20}{|c|}{ VALUES OF $m$} \\
\hline & \multicolumn{4}{|c|}{0} & \multicolumn{4}{|c|}{1} & \multicolumn{4}{|c|}{2} & \multicolumn{4}{|c|}{3} & \multicolumn{4}{|c|}{4} \\
\hline & $a$ & $b$ & $c$ & $d$ & $a$ & 8 & $c$ & $d$ & $a$ & $b$ & $c$ & $d$ & $a$ & $b$ & $c$ & $d$ & $a$ & $b$ & $c$ & $d$ \\
\hline 0 & 5 & 0 & 0 & 0 & 4 & 0 & 1 & 0 & 3 & 0 & 2 & 0 & 2 & 0 & 3 & 0 & 1 & 0 & 4 & 0 \\
\hline 1 & 4 & 1 & 0 & 0 & 16 & 4 & 4 & 1 & 12 & 3 & 8 & 2 & 8 & $\mathbf{2}$ & 12 & 3 & 4 & 1 & 16 & 4 \\
\hline 2 & 3 & 2 & 0 & 0 & 12 & 8 & 3 & 2 & 9 & 6 & 6 & 4 & 6 & 4 & 9 & 6 & 3 & 2 & 12 & 8 \\
\hline 3 & 2 & 3 & 0 & 0 & 8 & 12 & 2 & 3 & 6 & 9 & 4 & 6 & 4 & 6 & 6 & 9 & 2 & $\mathbf{3}$ & 8 & 12 \\
\hline 4 & 1 & 4 & 0 & 0 & 4 & 16 & 1 & 4 & 3 & 12 & 2 & 8 & 2 & 8 & 3 & 12 & 1 & 4 & 4 & 16 \\
\hline
\end{tabular}

NoTE.-The divisor, to obtain the interpolated value, is equal to the sum of the four multipliers of $a, b, c$, and $d$, which is always either $5\left(=\frac{1}{2}\right)$ or to $25\left(=\frac{1}{.04}\right)$.

This table is useful for reference, and in deducing interpolated values of any tabular function of two variables, where the given values, for both or for either of the variables, are tabulated at quinquennial intervals only. For example, the office rates for two-life assurances and for two-life annuities (whether joint, last survivor, or contingent) are frequently tabulated for every fifth age only of each of the two lives involved, and the ordinary process of deducing the interpolated values at intermediate ages, by taking proportionate parts of the differences of the given values of the function, is somewhat cumbersome, and requires care to obtain a correct result.

An example or two will make clear the practical application of the values given in the table:

(1). Given

$$
\begin{aligned}
& P_{30.40}=4 \cdot 433=a, \\
& P_{30.45}=5 \cdot 049=b, \\
& P_{35.40}=4 \cdot 688=c, \\
& P_{35,45}=5 \cdot 265=d .
\end{aligned}
$$

To find $P_{33,42}$. Here $m=3, n=2$, and we have, approximately,

$$
\begin{aligned}
& P_{38,4}=\frac{1}{2} \bar{s}(6 a+4 b+9 c+6 d) \\
& 6 \times 4.433=26.598 \\
& 4 \times 5 \cdot 049=20 \cdot 196 \\
& 9 \times 4 \cdot 688=42.192 \\
& 6 \times 5 \cdot 265=31.590
\end{aligned}
$$

the true value being 4.784 , 
By the ordinary method of differencing, and taking proportional parts, the process would be as follows:

$$
\begin{aligned}
& \mathrm{P}_{30.40}=4.433 \quad \mathrm{P}_{35.40}=4.688 \\
& \mathrm{P}_{30.45}=5.049 \quad \mathbf{P}_{35.45}=5.265 \\
& \Delta=+\cdot 616 \quad \Delta=+.577 \\
& \cdot 4 \Delta=+\cdot 246 \quad \cdot 4 \Delta=\cdot 231 \\
& P_{3042}=\overline{4 \cdot 679} \quad P_{3 \bar{s} .42}=\overline{4.919} \\
& \mathrm{P}_{30,42}=4.679 \\
& P_{3542}=4.919 \\
& \Delta=\overline{+\cdot 240} \\
& \cdot 6 \Delta=+\cdot 144 \\
& \mathbf{P}_{33.42}=4.823 \text { approximately. }
\end{aligned}
$$

(2). Required the approximate value, at date of purchase, of an annuity on the lives of a male aged 43 and a female aged 56 , according to the Government Life Annuity Experience, with interest at 3 per cent.

Here we have, from the recently published Institute Tables:

$$
\begin{aligned}
& a_{[56: 41]}=10 \cdot 948=a \\
& a_{[56: 466]}=10 \cdot 596=b
\end{aligned}
$$

also $m=0, n=2$, whence, approximately,

$$
\begin{aligned}
a_{[56: 43]} & =\frac{3}{5}(3 a+2 b) \\
3 \times 10 \cdot 948 & =32 \cdot 844 \\
2 \times 10 \cdot 596 & =21 \cdot 192
\end{aligned}
$$

$$
54: 036 \times \cdot 2=10 \cdot 807
$$

By the usual method of differences, the process would be

$$
\begin{aligned}
a_{[53: 41]} & =10 \cdot 948 \\
a_{[56: 46]} & =10 \cdot 596 \\
\Delta & =-\cdot 352 \\
4 \Delta & =-\cdot 141 \\
a_{[56 \cdot 43]} & =10 \cdot 807 \text { approximately. }
\end{aligned}
$$

There is here little to choose between the two methods, as regards labour, but the former process involves less liability to error from the use of signs, or the calculation of the proportionate parts.

The approximate values arrived at by interpolation to first differences only are, of course, not very aceurate. If second differences are introduced, the resulting formulæ are, in cases where 
$m$ and $n$ have both a significant value, somewhat cumbersome, as involving five tabular values of the function, and high multipliers. In the case, however (as in the recently published Government Annuity Tables) where $m=0$, that is, where the values for the one life are tabulated for every age, and for the other life at quinquennial intervals of age only, the formulæ and multipliers to second differences are more simple, and may be sometimes usefully employed where greater accuracy is required in the interpolated value.

For this purpose, alternative formulæ may be employed, the one, proceeding by ordinary differences:

$\phi_{x, y+n}=\phi_{x y}+\frac{n}{5}\left(\phi_{x . y+5}-\phi_{x y}\right)-\left(\frac{n}{10}-\frac{n^{2}}{50}\right)\left(\phi_{x . y+10}-2 \phi_{x y+5}+\phi_{x y}\right)$

$=\frac{1}{50}\left\{\left(50-15 n+n^{2}\right) \phi_{x y}+\left(20 n-2 n^{2}\right) \phi_{x . y+5}-\left(5 n-n^{2}\right) \phi_{x y+10}\right\}$.

the other, proceeding by central differences, as developed by Mr. Woolhouse, in his classical paper (J.I.A., xi, 61, 301) upon the subject of interpolation:

$\phi_{x . y+n}=\phi_{x y}+\frac{n}{5}\left(\phi_{x \cdot y+5}-\phi_{x y}\right)-\left(\frac{n}{10}-\frac{n^{2}}{50}\right)\left(\phi_{x y+5}-2 \phi_{x y}+\phi_{x . y-5}\right)$

$=\frac{1}{50}\left\{\left(50-2 n^{2}\right) \phi_{x y}+\left(5 n+n^{2}\right) \phi_{x y+5}-\left(5 n-n^{2}\right) \phi_{x \cdot y-5}\right\} \quad . \quad$.

It will be observed that by the formula (5) to ordinary second differences, the tabular values employed for deducing the interpolated value $\phi_{x . y+n}$ are $\phi_{x y}, \phi_{x . y+5}$, and $\phi_{x . y+10}$; while by the formula (6) to central second differences, the values employed are $\phi_{x . y-5}, \phi_{x y}$, and $\phi_{x, y+5}$.

For successive values of $n$ from 0 to 4 inclusive, the multipliers by formulæ (5) and (6) respectively, are shown in the following tables:

Ordinary Differences.

\begin{tabular}{|c|c|c|c|}
\hline${ }_{n}^{\text {Yal }}$ of & $\phi_{x . y}$ & $\phi_{x, y+5}$ & $\phi_{x . y+10}$ \\
\hline 0 & 1 & 0 & \\
1 & 18 & 9 & -2 \\
2 & 12 & 16 & -3 \\
3 & 7 & 21 & -3 \\
4 & 3 & 24 & -2 \\
\hline
\end{tabular}

Central Differenees.

\begin{tabular}{|c|c|c|c|}
\hline $\begin{array}{c}\text { Valnes of } \\
n\end{array}$ & $\phi_{x y-5}$ & $\phi_{x y}$ & $\phi_{x \cdot y+5}$ \\
\hline 0 & 0 & 1 & \\
\hline 1 & -2 & 24 & 3 \\
2 & -3 & 21 & 7 \\
3 & -3 & 16 & 12 \\
4 & -2 & 9 & 18 \\
\hline
\end{tabular}


In order to illustrate the relative closeness of the approximation, the several formulæ numbered (1), (5), and (6), may now be applied to the computation of the value of a joint-life annuity according to the $\mathrm{H}^{\mathrm{MI}} 3$ per-cent tables. Let it be required for example, to deduce approximate values of $a_{50.48}$, given the values of $a_{50.40}, a_{50.45}$, $a_{50.50}$ and $a_{50.55}$.

By formula (1) to first differences,

$$
\begin{aligned}
a_{50.48} & =\frac{1}{5}\left(2 a_{50.45}+3 a_{50.50}\right) \\
& =10.8331 .
\end{aligned}
$$

By formula (5) to second differences,

$$
\begin{aligned}
a_{50.48} & =\frac{1}{25}\left(7 a_{50.45}+21 a_{50.50}-3 a_{50.55}\right) \\
& =10.8578 .
\end{aligned}
$$

By formula (6) employing central second differences,

$$
\begin{aligned}
a_{50.49} & =\frac{1}{25}\left(16 a_{50.45}+12 a_{50.50}-3 a_{50.40}\right) \\
& =10.8543 .
\end{aligned}
$$

The true value, as given in the Institute $\mathrm{H}^{\mathrm{M}} 3$ per-cent tables, is 10.8535 ; and it will be seen that, as stated by Mr. Woolhouse (J.I.A., xi, 73 [note]), the central difference formula gives a more accurate result than that derived from the employment of ordinary second differences.

Addiscombe, Croydon,

$$
\begin{aligned}
& \text { I am, Sir, } \\
& \text { Yours, \&e., } \\
& \text { THOMAS G. ACKLAND. }
\end{aligned}
$$

30 December 189 อ.

\section{GRADUATION-MR. J. A. HIGHAM'S THEOREM.}

To the Eatitor of the Journal of the Institute of Actuaries.

SIR,-In the study of graduation formulas, I have been rather surprised that comparatively little use has been made of the very remarkable theorem given by Mr. J. A. Higham (J.I.A., xxv, 17 and 245-8), by which the result of any number of successive summations of the terms of a series is expressed by means of the first term of the series and its differences. Mr. Higham himself has fully explained the application of his theorem for purposes of graduation, and in his last contribution on the subject (J.I.A., xxxi, 319) he has shown how it may be made to yield Woolhouse's formula. The demonstration of the theorem which Mr. Higham gives seems to me unnecessarily difficult, and I therefore venture to submit the following simpler proof.

Suppose the terms of the series $u_{0}, u_{1}, u_{2}, \ldots u_{n}$ to be summed continuously in $p$ 's; the results to be summed in $q^{3} \mathrm{~s}$; the next results to be summed in $r^{2} \mathrm{~s}$, and so on till the series has been reduced to 